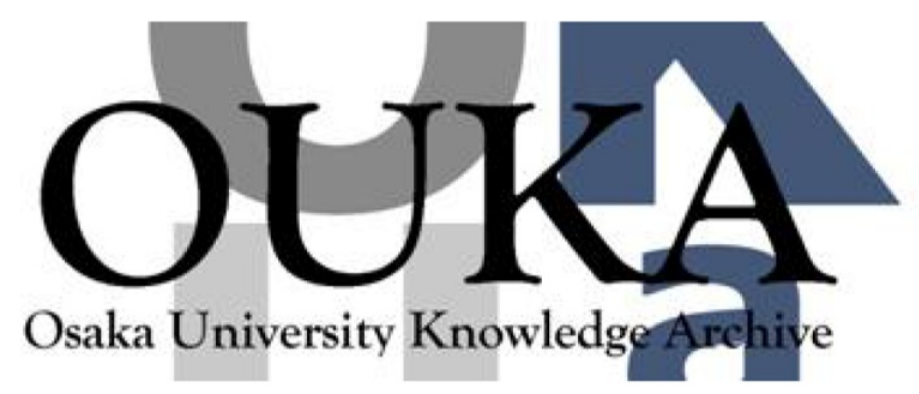

\begin{tabular}{|c|l|}
\hline Title & $\begin{array}{l}\text { NEUTRON SCATTERING MEASUREMENT IN CHROMIUM NEAR } \\
\text { THE NEEL TEMPERATURE }\end{array}$ \\
\hline Author(s) & Tsunoda, Yorihiko \\
\hline Citation & \\
\hline Issue Date & \\
\hline oaire:version VoR \\
\hline URL & https://hdl. handle. net/11094/30074 \\
\hline rights & \\
\hline Note & \\
\hline
\end{tabular}

Osaka University Knowledge Archive : OUKA

https://ir. Library. osaka-u. ac. jp/

Osaka University 
NEUTRON SCATTERING MEASUREMENT IN CHROMIUM NEAR THE NEEL TEMPERATURE

by

Yorihiko Tsunoda

Department of Physics, Osaka University

Toyonaka, Osaka 
Abstract

The magnetic properties of the metallic chromium can be interpreted in terms of itinerant electron model. The angular distribution of the neutron diffuse scattering around its 10 satellite reflection has been measured by double-axis spectrometer with the temperature range of $\mathrm{T}_{\mathrm{N}}-20{ }^{\circ} \mathrm{C} \leq \mathrm{T} \leq \mathrm{T}_{\mathrm{N}}+33^{\circ} \mathrm{C}$. Improvement of the instrumental resolution has made it possible to observe the correlation range more accurately. Correlation range of the spin system above the Néel temperature, which was analyzed from the experimental data on the basis of the ornsteinZernike type correlation function and the static approximation, has been obtained as $x_{I}^{2}\left(Q_{1 /}\right) \propto\left(T-T_{N}\right) 0.70 \pm 0.2$ and $x_{I}^{2}\left(Q_{\perp}\right) \propto$ $\left(T-T_{N}\right)^{0.54 \pm 0.2}$. The temperature dependence is remarkably smaller than those obtained in various substances and the theoretical conclusions of Ising or Heisenberg model.

Energy analysis of the scattered neutrons has also been measured by means of TOF method. The spin wave like collective excitations could, be observed above the Nél temperature, and the dispersion relation curve has been written clearly at $50^{\circ} \mathrm{C}$. The temperature dependence of the inelastic scattering intensities shows a critical feature with maximum value just above the Néel temperature. 
CONTENTS

\& 1. INTRODUCTION $\ldots \ldots \ldots \ldots \ldots \ldots \ldots \ldots \ldots \ldots \ldots \ldots \ldots \ldots \ldots$

§ 2. SAMPLE PREPARATION $\ldots \ldots \ldots \ldots \ldots \ldots \ldots \ldots \ldots \ldots \ldots \ldots \ldots \ldots$

\& 3. CRITICAL SCATTERING .................... 5

3 - a) Measurements ................... 5

3 - b) Temperature Control ................ 7

3 -c) Resolution Function ................. 7

3 - d) Experimental Data ................... 9

3 - e) Data Analysis ...................... 10

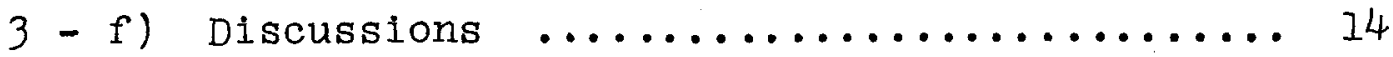

8 4. INELASTIC SCATTERING $\ldots \ldots \ldots \ldots \ldots \ldots \ldots \ldots \ldots \ldots$

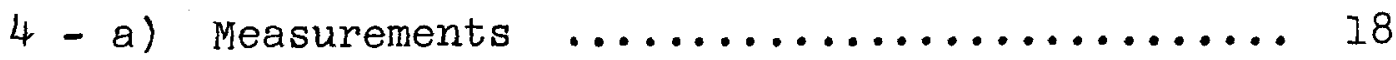

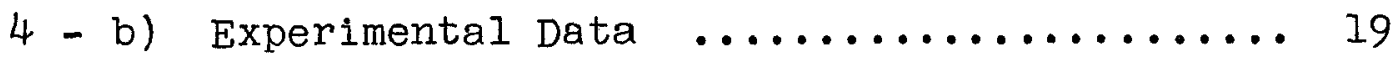

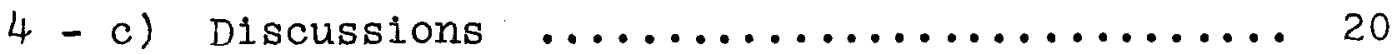

$\S 5$. CONCLUSTONS $\ldots \ldots \ldots \ldots \ldots \ldots \ldots \ldots \ldots \ldots \ldots \ldots \ldots \ldots \ldots$

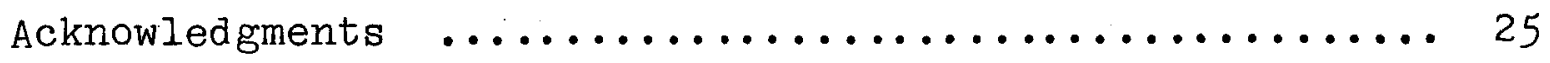

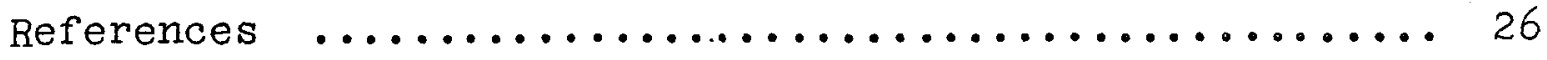

Figure Captions $\ldots \ldots \ldots \ldots \ldots \ldots \ldots \ldots \ldots \ldots \ldots \ldots \ldots \ldots$

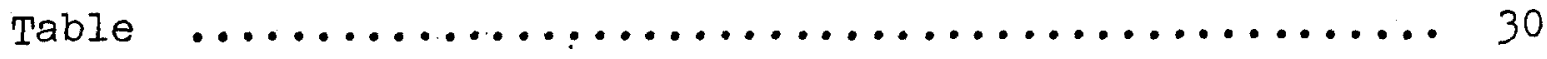

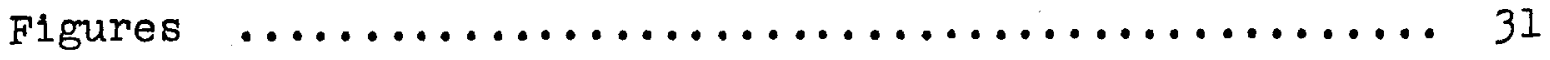




\section{§I. INTRODUCTION}

Metallic chromium is an antiferromagnetic substance with body centered cubic lattice, and the following experimental facts have been known about its magnetic properties. 1) In the neutron diffraction pattern, the satellite reflections corresponding to the long wave length sinusoidal magnetic structure are observed near the reciprocal lattice point. 2) The magnetic scattering intensity of neutrons suddenly vanishes at $38.5^{\circ} \mathrm{C}$ showing the first order transformation from sinusoidal phase to paramagnetic phase. 3) No trace of the paramagnetic scattering of neutrons is observed. 4) The Néel temperature and the period of the sinusoidal modulation are very sensitive to the impurities and strain. These magnetic properties have been interpreted by means of the spin density wave model which was formed through the exchange interaction of the itinerant electrons themselves.

The purpose of this experiment is to investigate the spin correlation of the spin density wave near the Neel temperature by using elastic and inelastic scattering of neutrons.

The spin behavior of chromium in paramagnetic phase was studied first by Wilkinson and his coworkers ${ }^{l}$ ). They measured the paramagnetic scattering of neutrons using chromium isotope without energy analysis and reported that they could not find the magnetic moment in paramagnetic phase within an experimental error. Mфller and his coworkers ${ }^{2}$, however, observed that the critical scattering in chromium persists up to $200^{\circ} \mathrm{C}$ in their double axis spectrometer method. The results ended to semiquantitative discussions because of the poor instrumental resolution and the imperfectness of the sample. Quite recently 
Als-Nielsen and Dietrich ${ }^{3)}$ analized the energy of scattered neutrons near the Neel temperature and found the magnetic excitation in spin density wave which was consistent qualitatively with the theory predicted by Fedders and Martin ${ }^{4}$.

We have measured also the magnetic critical scattering of neutrons by using double axis spectrometer ${ }^{5}$. Refined single crystal and improvement of the instrumental resolution make it possible to obtain more quantitative results. In the first half of this paper, we will report the results of the critical scattering measurements. In order to study the dynamical behavior of the spin system, the time of flight method has been used in the energy analysis of the scattered neutrons. The second half of this paper will be devoted to the inelastic scattering measurements. 
In order to get the high scattering intensity, the large volume sample is required for the experiment of the inelastic scattering of neutrons. While the magnetic properties of chromium depend very sensitively on the impurities and the lattice defects. The preparation of the chromium single crystal is one of the most troublesome works in this experiment.

The strain anneal method has been tried first to grow a chromium single crystal. Pure electrolytic chromium (99.999\% Johnson-Matthey) was melted in the arc furnace in argon atmosphere and was cut into several rods with the size of about $30 \times 7 \times 7 \mathrm{~mm}^{3}$. They were pressed uniaxially by the pressure of $1020 \mathrm{Kg} / \mathrm{mm}^{2}$ and annealed for a day in the siliconit furnace at the temperature of $15001600^{\circ} \mathrm{C}$. We have got the maximum grain of the volume about $8 \mathrm{~mm}^{3}$ which was far small to get enough the scattering intensities. Moreover, the previous experiment by Moller et al. 2) shows that the chromium single crystal prepared by strain anneal method is unsuitable for the critical scattering measurement because of s.trong effects of remaining strain.

As Arrott et al.6) have pointed out, the ideal strain free sample can be made from the vapor of chromium lodide. However, these maximum volumes are also less than about $100 \mathrm{~mm}^{3}$. As the results of various trials, we have found that the single crystals with suitable size grow by annealing the pure electrolytic chromium in the argon atmosphere at the temperature about $14000^{\circ} \mathrm{C}$ $1500^{\circ} \mathrm{C}$ for few hours.

our sample has the purity of $99.99 \%$ up on account of the Iimitation in the material volume, (By this method, the single 
crystal does not grow from the ingot which has once melted in arc furnace.) and the volume about $15 \times 15 \times 5 \mathrm{~mm}^{3}$. The ratio of resistance $\left(R_{\text {Room }} / R_{4.2}\right)$ is about 140 after annealing. It is difficult to prove whether this sample is strain-free or not. But as to speaking about impurities and lattice defects, the sample is considered to be good enough for the critical scattering measurement becase of high ratio of the resistance. Later, we will show experimentally that the sample shows the first order transition at Néel temperature, which is the best proof of the perfectness of the crystal. 
83. CRITICAL SCATTERING

The angular distribution of the critical magnetic scattering of neutrons by using double axis spectrometer gives us the information on the spacial distribution of the spin pair correlation function with same time near the Néel temperature.

3 - a) Measurements

Equipments used in our critical scattering measurement are the double-axis spectrometer established in JRR-2*. Their outline is illustrated in Fig. I.

For chromium, a satellite reflection near 100 reciprocal lattice point can be found corresponding to the sinusoidal modulation of the spin system, which gives us an advantageous condition to measure the weak peak such as critical scattering, because the magnetic scattering is separated completely from the nuclear one. But in the real single crystal, six satellite reflections are observed near 100 reciprocal lattice point due to the existence of the magnetic domains. Since these satellite positions exist very closely, it is difficult to separate even these elastic coherent scattering for some scanning direction. In the case of the diffuse scattering, the separation becomes poorer. In order to make better condition, fine collimator system (vertical 15', horizontal 30') has been used as shown in Fig. I at the sacrifice of the total scattering intensities.

* JRR-2; Japan Research Reactor No. 2 which is built at JAERI, Tokai, Ibaraki, Japan, is cp-5 type with the power of 10MW. 
Another troublesome problem comes from the higher order contamination of the neutrons. Though the nuclear reflection does not appear at the 100 reciprocal lattice point in chromium because of its b.c.c. crystal structure, since our monochromatic neutron beam includes the higher order neutrons of about 3\% (Copper single crystal was used as a monochromator) the neutrons with wave length of $\lambda / 2$ make a weak coherent peak of the 200 Bragg reflection at the 100 reciprocal lattice point. In critical scattering measurement, this peak makes a very serious problem. The intensity of the higher order component becomes comparable to the magnetic elastic scattering at the room temperature. In order to remove it, the $\mathrm{Er}_{2} \mathrm{O}_{3}$ filter with the thickness of $7 \mathrm{~mm}$ has been used. As erbium nucleus has a sharp resonance absorption at $460 \mathrm{meV}$, the incident neutron energy has been chosen at $114 \mathrm{meV}(0.852 \AA)$. The effects of the $\mathrm{Er}_{2} \mathrm{O}_{3}$ filter are shown in Fig. 2. The efficiency is estimated about $99.8 \%$ with the sacrifice of magnetic scattering intensity of $30 \%$. The most part of the remaining peak at the 100 reciprocal lattice point in Fig. 2 can be interpreted as the superposition of the $10 \delta$ and $10-\delta$ satellite reflections on account of its temperature dependence.

A satellite reflection corresponds to a spin density wave with single wave vector $Q$. If the spin density wave has anisotropy between its propagation airection and perpendicular to it, we shall be able to observe it by measuring the critical scattering distribution along the two principal lines, parallel and perpendicular direction to $Q$. The former corresponds to $\theta$-drive

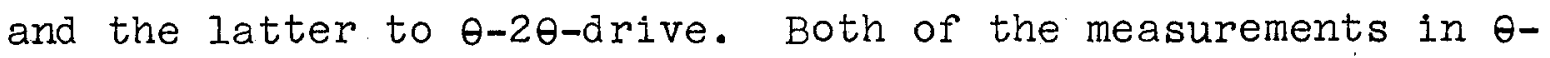


drive and in $\theta-2 \theta-d r i v e$ around the $1 \delta 0$ satellite point have been made. In the $\theta-2 \theta-d r i v e$, the incident neutrons with wave length of $0.998 \AA$ have been used to obtain higher intensities. We need not be worried about the higher order contamination, since the scattering vector does not go through on the 100 reciprocal lattice point in this scanning.

3 - b) Temperature control

Temperature control of the sample makes the important factor for the measurements of the critical phenomena. As the Néel temperature of chromium is near the room temperature, it is rather difficult to hold the sample at the constant temperature during the critical scattering measurement. The better condition has been found by using liquid nitrogen cryostat with a heater. Two copper-constantan thermocouples have been attached on the sample and used them for control and measurement of the temperature respectively. The temperature fluctuations of the sample have been less than $0.2^{\circ} \mathrm{C}\left(0.06 \%\right.$ of $\left.\mathrm{T}_{\mathrm{N}}\right)$. Uniformity of the temperature in the sample seems to be good enough because sample volume is far smaller than that of heat reserver. The critical scattering measurements have been done at several points between the room temperature and $72^{\circ} \mathrm{C}$.

3 - c) Resolution Function

Since the instrumental resolution in the neutron scattering is poorer than that in the case of $\mathrm{X}$-ray, it becomes a serious factor in discussing the line shape and the line width quantitatively.

If we write the resolution function $R(\underline{\underline{K}})$, the scattering intensity $I(\vec{k})$ is obtained from the following equation 


$$
I\left(\vec{K}^{\prime}\right)=\int_{-\infty}^{\infty} R\left(\vec{K}-\vec{k}^{\prime}\right) F(\vec{K}) d^{3} k
$$

where $F(k)$ is the scattering cross section expected when the ideal incident beam bursts the ideal sample, and $\vec{k}$ and $\vec{k} \cdot$ mean the arbitrary wave vectors in reciprocal lattice space. Thus the observable intensities are obtained from the convolution of the resolution function and the scattering cross section.

Experimental resolution depends on the mosaic spread of a monochromator and the sample, counter spread and collimator system. The method to calculate the resolution function has not been established. However, we can get directly the approximate form of the resolution function from the measurement of the Bragg reflection as proved by Als-Nielsen and Dietrich'). According to their way, two dimensional instrumental resolution function on the (001) plane was determined experimentally by scanning in two principal directions through the $1 \delta 0$ satellite point below the Néel temperature. As for the resolution function in the perpendicular direction to the above two, we have had to use the expected value from the collimator system because of the limitation in the experimental apparatus. Thus determined function is described approximately by the Gaussian function

$$
R\left(K_{x} K_{y} K_{z}\right)=\exp \left(-\frac{K_{x}^{2}}{\Gamma_{x}^{2}}\right) \exp \left(-\frac{K_{y}^{2}}{\Gamma_{y}^{2}}\right) \exp \left(-\frac{K_{z}^{2}}{\Gamma_{z}^{2}}\right)
$$

where $\Gamma_{x}=-.04589 \AA^{-1}, \quad \Gamma_{y}=0.005702 \AA^{-1}, \quad \Gamma_{z}=0.0114 \AA^{-1}, x$-axis is chosen to the $[100]$ direction and $y$-axis to the $\left(110^{\circ}\right]$ direction. 


\section{3 - d) Experimental Data}

Firstly the temperature dependence of the integrated intensities in 180 satellite peak has been measured and it shows first order phase transition between $38.0^{\circ} \mathrm{C}$ and $38.5^{\circ} \mathrm{C}$ as shown in Fig. 3. This indicates the perfectness of our sample because, as Arrott has shown, first order. phase transition at the temperature. of $38.3^{\circ} \mathrm{C}$ can be found only in the strain free chromium with very high purity.

The scattering intensities of neutrons measured at several temperatures above the Néel point are given in Fig. 4 and Fig. 5. Fig. 4 is the obtained intensities along Q-vector by $\theta$-driving. In order to get higher accuracy by increasing the statistics, the experimental values in the both sides of the 100 reciprocal lattice point, through $1 \delta 0$ point and $1-\delta 0$ point, have been summed up. This treatment is reasonable, because the same magnetic domain contributes to these reflections. The scattered intensities obtained by $\theta-2 \theta-d r i v i n g$ (the perpendicular direction to the Qvector) are given in Fig. 5. The intensive counts are due to using the longer wavelength incident neutrons of $0.998 \AA$. The observed peaks scanned along the $Q$-vector for some points below the Neel temperature are indicated in Fig. 6. This figure also shows the abrupt change of the intensities between $38^{\circ} \mathrm{C}$ and $39^{\circ} \mathrm{C}$ corresponding to the first kind phase transformation.

These observed patterns have the following characteristics of the critical scattering. I) Even just above the Néel temperature, the peak intensity is very weak. (less than $10 \%$ of the magnetic coherent peak at $\left.0.97 \mathrm{~T}_{\mathrm{N}}\right)$ 2) The scattered intensities obtained at off-Bragg point have a maximum value just 
above the Néel temperature. 3) The scattered intensities decrease rather rapidly below the Néel temperature. 4) The line shapes become diffuse as the temperature increase.

From these results, we can make the conclusion that the diffuse peaks obtained near the Néel temperature are the critical magnetic scattering, and that the critical scattering intensities distribute around the satellite position. High intensities at 100 reciprocal lattice point can be interpreted as the superposition of the tails of the diffuse peaks at each satellite position.

All the experimental data are subtracted by the back ground counts determined from the measurement at the point apart from 100 lattice point by $15^{\circ}$ where the critical scattering was already vanishing.

3 - e) Data Analysis

Since our magnetic system is very complicated, the easy treating method has been used in analyzing the data. The observed data below the Néel temperature, in which we have few theoretical predictions, have been taken out from our discussions because the phenomena become more complex.

In order to make the correction about the instrumental resolution, the scattering cross section which calculated theoretically under the appropriate assumptions is usually used, because the direct evaluation method of the true scattering cross section from the measured intensities has not been established yet. The scattering cross section of neutrons for the itinerant electron model has been given by Izuyama, Kim and Kubo ${ }^{81}$. The differential cross section can be described by the imaginary part of the wave 
vector-frequency dependent magnetic susceptibility $\chi$ (qw) as a result of the fluctuation dissipation theorem. Above the transition temperature, $\chi(q w)$ may be written by the following expression under the random phase approximation.

$$
\chi(q \omega)=\Gamma(q \omega) /[1-V \Gamma(q \omega)]
$$

where $(q w)$ is the susceptibility of noninteracting electrons and $\mathrm{V}$ is the matrix element of the electron interaction potential. The factor $I /\left[I-V\left[\left(\begin{array}{ll}0 & 0\end{array}\right)\right]\right.$ denotes the stoner enhancement factor due to the electron exchange interaction. According to the calculation under the reasonable assumptions of $v_{F} q$ shw and $q / k_{F} \ll I$, where $v_{F}$ and $k_{F}$ mean the electron velocity and wave vector with the Fermi energy respectively, and $\hbar \omega$ is the energy change of neutrons in the scattering process, the cross section for the angular distribution of the critical scattering in the vicinity of a reciprocal lattice point is given by the equation

$$
F(\vec{q})=\frac{d \sigma}{d \Omega}=\left(\frac{2 \gamma e^{2}}{m c^{2}}\right)^{2}|f(\vec{B})|^{2} \frac{c}{\hbar_{1}^{2}+q^{2}}
$$

$\gamma=1.91 ;$ neutron magnetic moment

$$
\text { e; electron charge } \mathrm{m} \text {; electron mass }
$$

$f(\vec{B})$; magnetic form factor.

and

$x_{1}$; inverse correlation range.

This expression has the same form as derived by van Hove ${ }^{9}$ using ornstein-zernike type correlation function.

$$
\langle S(0) S(R)\rangle=\frac{S(S+1)}{4 \pi r_{1}^{2}} \exp (-\gamma, R) / R
$$

Where $S(O)$ and $S(R)$ equal to the magnitude of the spins at origin and position $R, x_{I}$ denotes the parameter describing the spin 
correlation range and $\mathrm{C}$ is a parameter closely related to the correlation strength $\gamma_{I}$. These are slowly varing with the temperature. The temperature dependence of $x_{I}$ is calculated theoretically from the stand point of the statistical mechanics. The high temperature expansion gives the relation $x_{1}{ }^{2} \propto\left(\mathrm{T}-\mathrm{T}_{\mathrm{C}}\right)^{1.33}$ for the body centered cubic lattice in Heisenberg model. When the neutron energy change in scattering process is far smaller than the incident neutron energy, this expression becomes good approximation to the critical scattering. In other words, the system under the critical slowing down should not have rapid time variation during the neutron go through the sample. This assumption is known as "static approximation".

Six kinds of the magnetic domains corresponding to the various $Q$ and spin directions can exist in the substance with the transverse sinusoidal magnetic structure and with the cubic crystallographic structure. All these six satellite points appearing near 100 reciprocal lattice point have been considered in this calculation, because each peak becomes diffuse and their tails affect each other on their peak intensities at the critical temperature.

Assuming $F(\vec{q})$ as a Iorentzian according to the equation (4), the expectable intensity can be calculated as a convolution of the resolution function (2) by using equation (1).

In the calculation, several assumptions have been made. 1) Static approximation -- The cross section with the form of equation (4) is derived from the static approximation for the ferromagnetic substance, but here, it is also assumed to be valid for antiferromagnetic substance if the $\vec{q}$-vector is measured from the magnetic super lattice point. 2) The equivalent distribution 
of the magnetic domains -- In analyzing the data, we have made an assumption that six magnetic domains distribute with the equal probability. 3) The temperature dependence of the sateliite position -- The temperature dependence of the period in the spin density wave has been measured below the Néel temperature by Shirane and Takeilo). The extrapolated values of their results have been used as the satellite position above the Néel temperature. 4) Magnetic form factor -- The obtained intensities by scanning along the scattering vector $(\theta-2 \theta-d r i v e)$ make the antisymmetric patterns due to the magnetic form factor. The correction for the magnetic form factor has been made by using the data of Moon et al. 11)

The expectable intensities have been calculated under these assumptions. For example, the equation which should be calculatea for the $\theta$-arive is the following.

$$
\begin{aligned}
& I\left(q_{x}=0 . q_{y} q_{z}=0\right)=\iiint_{-\infty}^{\infty} \int e^{-\left(\frac{K_{x}^{2}}{\Gamma_{x}^{2}}\right)} e^{-\frac{\left(q_{y}-K_{y}\right)^{2}}{\Gamma_{y}^{2}}} e^{-\left(\frac{K_{z}^{2}}{\Gamma_{z}^{2}}\right)} \\
& \times C\left\{\frac{1}{x_{y}^{2}+\left[K_{x}^{2}+\left(q_{y}-K_{y}\right)^{2}+k_{z}^{2}\right)}+\frac{1}{K_{y}^{2}+\left[K_{x}^{2}+\left(2 Q-q_{y}-K_{y}\right)^{2}+K_{z}^{2}\right]}\right. \\
& +\frac{2}{K_{1}^{2}+\left[\left(K_{x}-Q\right)^{2}+\left(Q+q_{y}-k_{y}\right)^{2}+K_{z}^{2}\right]}+\frac{2}{K_{1}^{2}+\left[\left(K_{x}+Q\right)^{2}+\left(Q+q_{y}-K_{y}\right)_{T}^{2} K_{z}^{2}\right]} \\
& \left.+\frac{1}{\alpha_{y}^{2}+\left[K_{x}^{2}+\left(Q_{1}+q_{y}-K_{y}\right)+\left(Q+K_{z}\right)^{2}\right]}+\frac{1}{\gamma_{y}^{2}+\left(K_{x}^{2}+\left(Q+q_{y}-K_{y}\right)^{2}+\left(Q-K_{z}\right)^{2}\right]}\right\} \\
& x d k_{x} d k_{y} d k_{z} .
\end{aligned}
$$


Where $Q$ means the wave vector of spin density wave measured from 100 point and its value is known as a function of the temperature in advance as we mentioned above. $x_{1}$ and $C$ are the parameters with the temperature dependence. Calculations were carried out by using the computer NEAC 2200 and the trial and error fit between the experimental and calculated values was made by adjusting two parameters $x_{I}$ and $C$. The best fit curves along $Q$-parallel and Q-perpendicular directions are arawn in Fig. 4 and Fig. 5 by solid lines respectively. The parameters $x_{I}$ and $c$ determined by this method are listed in Table 1 .

If we write the temperature dependence of $x_{1}^{2}$ as $x_{1}^{2} \propto\left(T-T_{N}\right)^{\gamma}$ according to the scaling law, $\gamma$ can be determined from the slope of the $x_{1}{ }^{2}$ versus $\left(T-T_{N}\right)$ diagram in log-log scale as shown in Fig. 7. The results can be represented approximately

$$
\begin{aligned}
& x_{I}^{2}\left(Q_{I}\right) \propto\left(T-T_{N}\right) 0.70 \pm 0.2 \\
& x_{I}{ }^{2}\left(Q_{\perp}\right) \propto\left(T-T_{N}\right) 0.54 \pm 0.2
\end{aligned}
$$

These $\gamma$-values are distinctiy small compared with the various theoretical models and the experimental valus hitherto obtained.

Izuyama theory predicts that the parameter $C$ is proportional to $\mathrm{T}$. $\mathrm{C}\left(\mathrm{T}_{\mathrm{N}}\right)$ versus $\mathrm{C}(\mathrm{T})$ values are also listed in Table 1. The agreement is fairly well.

The differential cross section incluaing a $q^{4}$ term was also calculated, but the agreement with the experiemntal data became rather poor.

3 - f) Discussions

The distinguishable points of this experiment are the 
improvement of the instrumental resolution and making use of the refined sample, and they made it possible to make more quantitative discussions on the critical scattering of chromium. Although the magnetic properties of chromium can be well explained in terms of the itinerant electron model and its magnetic order is formed by the first order phase transformation, the critical fluctuations around the magnetic super lattice point can be found. The intensity of the critical scattering has also Iorentzian distribution in the reciprocal lattice space. The spin correlation range $x_{1}^{-1}$, which should be understood by the exchange enhancement factor in the case of the itinerant magnetic systems, is estimated about $50 \AA$ just above the Néel temperature and this is almost same value with that of iron ${ }^{22}$ )I3)I4).

In contrast to the Móllers experiment, an anisotropic property of $x_{1}$ with respect to the propagation direction of the sinusoidal modulation has been found in our experiment. The ratio of the correlation range $e$ (where $e=x_{I}(Q /) / x_{I}(Q \perp)$ ) is approximately 4/3. Such an anisotropy was already found in terbium by Arrott et al. 15 )

While $\gamma$-values are the smallest of all kinds of magnetic substances which have ever been measured. It is dangerous to jump at a conclusion that the small temperature dependence of $x_{1}$ should be reflex of the itinerancy in chromium magnetic electrons, because in nickel which is considered to be a typical example of an itinerant ferromagnetic substance, the consistent experimental value with the Heisenberg modei has been obtained ${ }^{16}$ ). We can consider several possibilities that the conventional critical scattering analysis using ornstein-zernike type correlation 
function may be unsuitable for chromium. First, it is a question whether the Izuyama theory which based on the simplified model with single band can be applied directly to chromium with a complicated band structure 17)I8). Moreover, we have used the value of $38.5^{\circ} \mathrm{C}$ as a Neel temperature in our data analysis. This value does not coincide with the paramagnetic Néel temperature because of the first kind phase transition. Then, the revaluation of $\mathbb{T}_{N}$ and $\gamma$ values have been tried from observed inverse correlation ranges by using the least mean square method, since it might be considered that we have measured the skirt of the critical scattering occurring near the paramagnetic Neel temperature. Results have been given as follows.

$$
\begin{array}{ll}
T_{N}^{\prime}\left(Q_{\text {II }}\right)=36.8^{\circ} \mathrm{C} & \gamma^{\prime}=0.84 \\
T_{N}^{\prime}\left(Q_{\perp}\right)=38.3^{\circ} \mathrm{C} & \gamma^{\prime}=0.54
\end{array}
$$

Revaluated $\gamma$-values are still small compared with the values which have ever been measured. The last problem comes from the doubie axis spectrometer method for critical scattering measurement. All the neutrons which were scattered into $2 \theta-\alpha i r e c t i o n$ both with and without energy change are counted irrespectively in this method. If the spin correlation has a short life time above the Néel temperature compared with the time that neutrons pass through the system, the critical scattering will contain the neutrons with high inelasticity and the static approximation which we have used will break down. In order to remove this problem, we shall have to analyze the energies of scattered neutrons. Thus, the inelastic scattering measurement in chromium might give us some solutions, at least for the last problem. 
\$4. INELASTIC SCATTERING

From the measurements of the inelastic scattering of neutrons, some informations about the space and time correlation of the spin system can be known. Usually, spin motion above the transition temperature is treated by the simplified spin diffusion model, which stands on the assumption that the fluctuation occurred at the origin at the time $t=0$, prevails to the point $r$ and time $t$ by following the same kind of probability function as an atomic diffusion.

According to this model, the scattering cross section is expressed by the Lorentzian function in energy space with the half-value width $\Gamma$.

$$
\begin{gathered}
\frac{d^{2} \sigma}{d \Omega d \omega}=A\left(\vec{k} \vec{k}^{\prime}\right) \sum_{\alpha \beta}\left(\delta_{\alpha \beta}-\hat{k}_{\alpha} \hat{k}_{\beta}\right) \frac{T}{T_{c}} \frac{1}{r_{1}^{2}\left(K_{1}^{2}+q^{2}\right)} \frac{\Gamma}{\omega^{2}+\Gamma^{2}} \\
A\left(\vec{k} \vec{K}^{\prime}\right)=\left(\frac{2 e^{2} \gamma}{m i c^{2}}\right) \frac{2 N}{\pi \hbar} \frac{S(S+1)}{3 k_{B}} \frac{k^{\prime}}{K}|f(\vec{B})|^{2}
\end{gathered}
$$

where $\widehat{K}$ denotes the unit vector of the scattering vector direction, $\alpha$ and $\beta$ mean the cartesian coordinate axes $x, y$ and $z \cdot{ }^{T_{C}}$ is a transition temperature. $\quad \Gamma$ is described as

$$
I=11^{2}
$$

by using the simple diffusion model with a phenomenological spin diffusion constant 1 . Thus, in the measurement of the inclastic scattering of neutrons at the critical temperature, we can expect the broadened distribution of the scattered neutron energies 
corresponding to the diffusion mode of the spin system.

4 - a) Measurements

For the purpose of the inelastic scattering measurement, the triple rotors spectrometer installed on JRR-2 has been used. The instruments are illustrated briefly in Fig. 8. Three rotors rotate with a constant speed and with a proper phase difference. The first and the third rotors produce pulsed monochromatic neutrons and the second is to remove the lower order contaminations. The neutron energies after scattered by the sample inelastically are analyzed by TOF method. The incident neutron energy of 8.2 meV $(3.16 \AA)$ has been used in our measurement. Energy resolution depends on some factors such as incident neutron energy, rotor speed, width of slit, flight path and so on. Our rotors have a revolution rate of 10000 R.P.M. and the energy resolution of $8 \%$.

At first, to investigate the spin diffusion mode around the $1 \delta 0$ point, the crystal was set so that the scattered neutron wave vector passed through the 150 point in the (001) plane. Where the $1 \delta 0$ point above the Néel temperature was determined by extrapolating the temperature dependence curve of the satellite point below the Néel temperature. In this setting, the momentum transfered from or to neutrons nearly orients [110] direction. The observed intensities with several different temperatures are shown in Fig. 9. In this figure, the left and right sides of the central peak correspond to the energy gain and loss of neutrons by scattering process respectively. In spite of having taken about 8 hours to obtain one curve, the intensities were very weak at high temperature. Then, the data were made the two point smoothing to get high statistics. The uniform back ground and 
incoherent elastic component were already subtracted. (Back ground counts were determined from the arithmetic average of the count numbers in a hundred channels.) An error of the time of flight due to the fluctuation of the control in rotor system is within $20 \mu \mathrm{sec}$.

The observed spectra were consisted of two groups of the scattered neutrons. The central intense peak with large temperature dependence can be ascribed to the critical quasi-elastic scattering. Instead of the drastic line broadening, small but distinct reflection appeared at the left side of the central peak at each temperature. Since the side peak does not depend on the temperature so strongly as the central peak, it is considered not to be the subordinate image of the central peak due to the instrumental origins. We can conclude that these subpeaks are due to some inelastic scattering with the energy change of $0.7 \sim 0.8 \mathrm{meV}$. In order to clarify the origin of these inelastic subpeaks, the setting of the sample was changed so that the incident and scattered neutron wave vectors lay nearly perpendicular to the 001 axis, and that the scattered neutron wave vectors pass through the 10 satellite point at all time. (See Fig. II) The effects of other satelites can be reduced by this setting, and are negligible for the determination of $w-q$ relation. About 8 hours have been devoted to each measurement. The temperature control of the sample was just the same as the case of critical scattering measurement.

4 - b) Experimental data

An example of the time of flight spectrum is shown in Fig. 10. In this figure, the intensive peak on the right hand side is 
elastic incoherent one. We can observe the inelastic peak separatedly on the left hand side of the elastic incoherent peak. From the energy and momentum conservation law, the energy and the momentum changes of the neutrons can be determined.

Several time of flight spectra measured at $500 \mathrm{C}\left(1.037 \mathrm{~T}_{\mathrm{N}}\right)$ with different inelastic peaks are given in Fig. 11. Undoubtedly these spectra show the existence of the collective excitation mode having some dispersion relation. We have drawn the dispersion relation curve of this collective excitation in chromium above the Néel temperature clearly as shown in Fig. 12. In these measurements, the directions of the scattered neutrons are not exactly the same, but lie very close to each other, so that these patterns can be analyzed as obtained in the same direction of q-vector. Where qvector means the wave vector of the collective excitation mode and is measured from the $10 \delta$ point. Large error bars are ascribed to the focussing effect in obtaining intensive inelastic peaks. The temperature dependence of the inelastic peak intensities has been also measured at several points between $71{ }^{\circ} \mathrm{C}\left(1.037 \mathrm{~T}_{\mathrm{N}}\right)$ and $23.2{ }^{\circ} \mathrm{C}$ $\left(0.951 \mathrm{~T}_{\mathrm{N}}\right)$. During this measurement, the crystal setting was not changed and the energy gain of the scattered neutrons was $4.3 \mathrm{meV}$ at $50^{\circ} \mathrm{C}$. The examples of observed spectra and the integrated intensity is found just above the NGel temperature and rapid decrease of the integrated intensities is observed as the temperature decreases. The same phenomena are also found for the intensities of the side peaks of Fig. 9. The temperature variation of the dispersion curve has not been observed between $50^{\circ} \mathrm{C}$ and $23.2^{\circ} \mathrm{C}$ within the experimental error.

4 - c) Discussions 
The inelastic scattering measurements have thrown us a new problem. The unexpected collective excitation mode with the dispersion relation has been observed near the Neel temperature. But this does not mean that the spin diffusion type damping was not found in this spin system. Fig. 9 shows rather the coexistence of the diffusive mode and the collective excitation mode. We have tried to analyze the main peaks in Fig. 9 under the simple diffusion approximation like eq. (7) with the parameter. Where the energy and space resolution of the instrument was corrected and the determined values by double-axis spectrometer method were used as the inverse correlation ranges. But the line shape did not depend so strong on $\mathcal{\Lambda}$ and it was rather more sensitive for $x_{1}$. Maybe, the reason is considered to be due to the poor energy resolution of our instruments. Moreover, since the observed spectra have unresolved subpeaks, we had to give up the quantitative discussions about the diffusion coefficients. Because in the time of flight method, not the point but some finite region on the line of the scattered neutron wave vector in the reciprocal lattice space contributes to the scattering simultaneously, it is very difficult to measure the line broadening corresponding to the spin diffusion mode. More accurate experiments using triple-axis spectrometer are desired to investigate the diffusive mode in chromium. The dispersion relation curve has the slope of $46 \pm 15$ mevA and nearly coincides with the longitudinal phonon dispersion curve to the $[110]$ direction. However, this collective excitation mode can be considered to be a magnetic origin from the following reasons. I) The magnetic super lattice point $10 \delta$ is an original point of this dispersion curve. 2) The temperature dependence 
of the integrated intensities shows the maximum value just above the Neel temperature. Though the magneto-vibrational mode with the same dispersion relation as that of phonon can be anticipated below the Neel temperature, it must vanish at the Neel temperature and above. In nickel, Komura, Lowde and windsor have found the spin wave like peaks with the maximum intensity near the Curie temperature ${ }^{19)}$. These cannot be explained by simple paramagnons in the critical region. Our results bear some resemblance to them on the temperature dependence of the integrated intensities. Quite recently, magnetic excitation in the spin density wave has been observed near the Neel temperature by Als-Nielsen and Dietrich ${ }^{3)}$. The slope of their dispersion relation curve is so steep that the peaks around the $\delta 10$ satellite point-are unresolved from the limitation of the spectrometer resolution. And their results are qualitatively consistent with the prediction by Fedders and Martin. Our obtaining spectra show the different dispersion relation which is rather similar to that expected in a usual antiferromagnetic substance with linear dependence in small q-region. And the peak corresponding to steep dispersion relation can hardly be found in our experimental condition shown in Fig. 11 . Though we have no definite answer to explain these discrepancies between Nielsen's data and ours, two probable solutions can be considered. One of them is ascribed to the anisotropy of dispersion relation, since they have measured along 100 direction and the present along nearly 110. Another solution is that the entirely different excitations have been measured by each other and our excitation mode may be due to a new collective excitation occurring when the spin density wave become unstable. The inelastic scattering measurements in chromium are not completed in this 
stage, but propose the new problems about the spin dynamics in the spin density wave. Further measurements at extended temperature range and with the different crystal setting are much desired. 


\section{Conclusions}

The angular distribution of the critical magnetic scattering of neutrons has been observed in terms of the double-axis spectrometer in metallic chromium near the Neel temperature. The correlation range is estimated about $50 \AA$ just above the Néel temperature and the temperature dependences of inverse correlation ranges are obtained as $\kappa_{1}{ }^{2}\left(Q_{11}\right) \propto\left(T-T_{N}\right)^{0.70 \pm 0.2}$ and $\kappa_{1}{ }^{2}\left(Q_{\perp}\right) \propto$ $\left(\mathrm{T}-\mathrm{T}_{\mathrm{N}}\right) 0.54 \pm 0.2$

In the measurements of the inelastic scattering of neutrons, spin wave like collective excitation has been found above the Néel temperature. The dispersion relation curve with a slope of $46 \pm 15$ meVA has been obtained at $50^{\circ} \mathrm{C}\left(1.037 \mathrm{~T}_{\mathrm{N}}\right)$. The inelastic scattering peaks have a critical feature in their intensities. In any case, the strong dynamic fluctuations are persistent at the paramagnetic phase in metallic chromium. It is quite probable that the critical scattering measured by the double axisspectrometer includes some inelastic coherent scattering as revealed by Fig. 9. Small temperature dependence of $\kappa_{1}{ }^{2}$ might be due to the contamination of this inelastic coherent scattering. We may conclude that the data analysis in terms of the static approximation in critical scattering is unsuitable for chromium because of the strong inelasticity of the scattered neutrons near the Néel temperature. 


\section{ACKNOWLEDGMENTS}

The author is much indebted to Professor N. Kunitomi for kindest guidance and many valuable discussions during the course of this investigation.

He would like to thank Dr. Y. Hamaguchi, Senior Scientist in Japan Atomic Energy Research Institute, for supervising him throughout this experiment.

The author also expresses his gratitude to Dr. T. Yamada for cooperation of the sample preparation and to Dr. Y. Yamada and $Y$. Nakai for their continual interest and encouragement. 
1) M.K. Wilkinson, E.O. Wollan, W.C. Koehler \& J.W. Cable Phys. Rev. 127 (1962). 2080.

2) H.B. Mфller, K. Blinowski, A.R. Mackintosh \& T. Brun Solid State Commun. 2 (1964) 109.

3) J.Als-Nielsen \& O.W. Dietrich Phys. Rev. Letters 22 (1969) 290.

4) P.A. Fedders \& P.C. Martin Phys. Rev. 143 (1966) 245.

5) Y. Hamaguchi, Y. Tsunoda \& N. Kunitomi J.Appl. Phys. 39 (1968) 1227.

6) S.A. Werner, A. Arrott \& H. Kendrick Phys. Rev. 155 (1967) 528.

7) J.Als-Nielsen \& O.W. Dietrich Phys. Rev. 153 (1967) 706 .

8) T. Izuyama, D.J. Kim \& R. Kubo

J. Phys. Soc. Japan 18 (1963) 1025.

9) L. Van Hove Phys. Rev. 95 (1954) 1374.

10) G. Shirane \& W.J. Takei

J. Phys. Soc. Japan 17 Suppl. B-3 (1962) 35.

11) R.M. Moon, W.C. Koehler \& A.L. Trego J. Appl. Phys. 37 (1966) 1037 .

12) L. Passel, K. Blinowski, T. Brun \& P. Nielsen Phys. Rev. 139 (1965) A1866.

13) M.F. Collins, R. Nathans, L. Passel \& G. Shirane Phys. Rev. Letters 21 (1968) 99. 
14) M.F. Collins, V.J. Minkiewicz, R. Nathans, L. Passel \& G. Shirane to be published in Phys. Rev.

15) A. Arrott, S.A. Werner, M.J. Cooper, R. Nathans, \& G. Shirane J. Appl. Phys. 38 (1967) 969.

16) M.F. Collins, V.J. Minkiewicz, R. Nathans, L. Passel \& G. Shirane to be published

17) W.M. Lomer Proc. Phys. Soc. (London) 80 (1962) 489.

18) T.L. Loucks Phys. Rev. 139 (1965) Al181.

19) S. Komura, R.D. Lowde \& C.G. Windsor Reports in Symposium on Neutron Inelastic Scattering IAEA Copenhagen 1968. 


\section{FIGURE CAPTIONS}

Figure 1. Double-axis spectrometer installed in JRR-2. $\mathrm{Er}_{2} \mathrm{O}_{3}$ filter is placed between the third collimeter and the $\mathrm{BF}_{3}$-counter.

Figure 2. Efficiency of Er-filter. The solid line and the dashed line show the observed intensities without and with the filter respectively. The measurement has been done by $\theta-$ drive at $-53^{\circ} \mathrm{C}$. Small peak at the center of dashed Iine is ascribed to the tails of $10 \delta$ and $10-\delta$ satellite peaks.

Figure 3. Temperature dependence of $1 \delta 0$ satelite reflection. The abrupt intensity change can be found between $38^{\circ} \mathrm{C}$ and $38.5^{\circ} \mathrm{C}$.

Figure 4. The temperature dependence of the scattered intensities scanning in the direction of $Q_{/ /}$. The solid Ines show the best fit curves calculated from the convolution of the Lorentzian function and the resolution function.

Figure 5. The temperature dependence of the scattered intensities scanning in the direction of $Q \perp$. The solid Ines show the best fit calculated curves.

Figure 6. The scattered intensities below the Néel temperature. For the sake of the comparison, the experimental data just above the Néel temperature are plotted.

Figure 7. The temperature variation of the inverse correlation ranges in log-log scale. The dashed line shows the relation $\kappa_{1}^{2} \propto\left(\mathrm{T}-\mathrm{T}_{\mathrm{N}}\right)^{1.33}$. 
Figure 8 . Outline of the triple rotors spectrometer settled in JRR-2 .

Figure 9. The temperature variation of the time of flight spectra. Each spectrum consists of two groups of scattered neutrons, intense peak centered at the elastic position and subpeak with small temperature dependence.

Figure 10. The typical example of the time of flight spectrum obtained above the Néel temperature in chromium. The left hand side of the elastic incoherent peak corresponds to the neutron energy gain from the system. The magnetic excitation peak is found at the left side of the elastic incoherent peak.

Figure 11. a) Explanation of the crystal setting. The scattered neutron wave vectors always pass through the $10 \delta$ point. b) Some examples of observed spectra at $50^{\circ} \mathrm{C}$ with various energy changes. Right hand side peaks are elastic incoherent one and the arrows show the collective excitations.

Figure 12. Dispersion relation observed in chromium at $50^{\circ} \mathrm{C}$ $\left(1.037 \mathrm{~T}_{N}\right) . \overline{\mathrm{q}}$-vector is measured from the $10 \delta$ point.

Figure 13. a) The temperature dependence of the integrated intensities of the collective excitation peaks. The, crystal setting was not changed during these measurements. b) Some examples of the observed spectra. 


\begin{tabular}{|c|c|c|c|c|c|}
\hline $\begin{array}{r}\mathrm{T}{ }^{\circ} \mathrm{C} \\
-\quad \\
\end{array}$ & $39^{\circ} / \mathrm{T}$ & $\begin{array}{l}K_{1}\left(Q_{\prime \prime}\right) \\
\left(10^{-2} \AA^{-1}\right)\end{array}$ & $C\left(39^{\circ}\right) / C(T)$ & $\begin{array}{c}K_{1}\left(Q_{\perp}\right) \\
\left(10^{-2} \AA^{-1}\right)\end{array}$ & $C\left(39^{\mathrm{c}}\right) / \mathrm{C}(\mathrm{T})$ \\
\hline 39.0 & 1 & $1.4 \pm 0.2$ & 1 & $1.1 \pm 0.2$ & 1 \\
\hline 42.0 & 0.991 & $1.9 \pm 0.2$ & 0.96 & & \\
\hline 42.5 & 0.989 & & & $1.8 \pm 0.2$ & 0.99 \\
\hline 45.0 & 0.981 & $2.5 \pm 0.2$ & 0.97 & & \\
\hline 49.5 & 0.967 & $2.8 \pm 0.3$ & 0.93 & $2.2 \pm 0.3$ & 0.95 \\
\hline 60.0 & 0.937 & $3.8 \pm 0.4$ & 0.94 & $2.6 \pm 0.3$ & 0.96 \\
\hline 72.0 & 0.904 & $4.4 \pm 0.5$ & 0.94 & $3.3 \pm 0.3$ & 0.91 \\
\hline
\end{tabular}

Table 1 


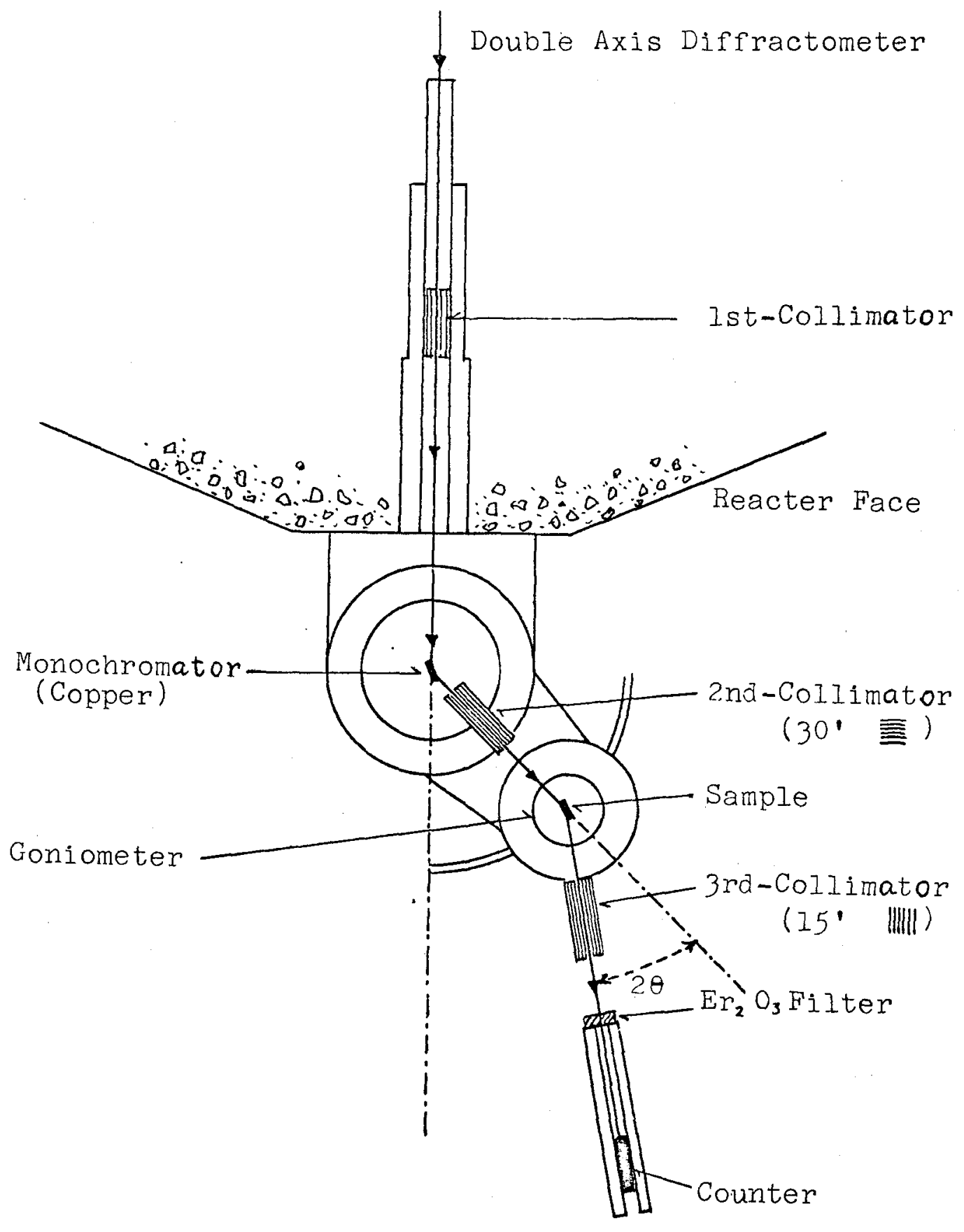

Fig 1

$-31-$ 


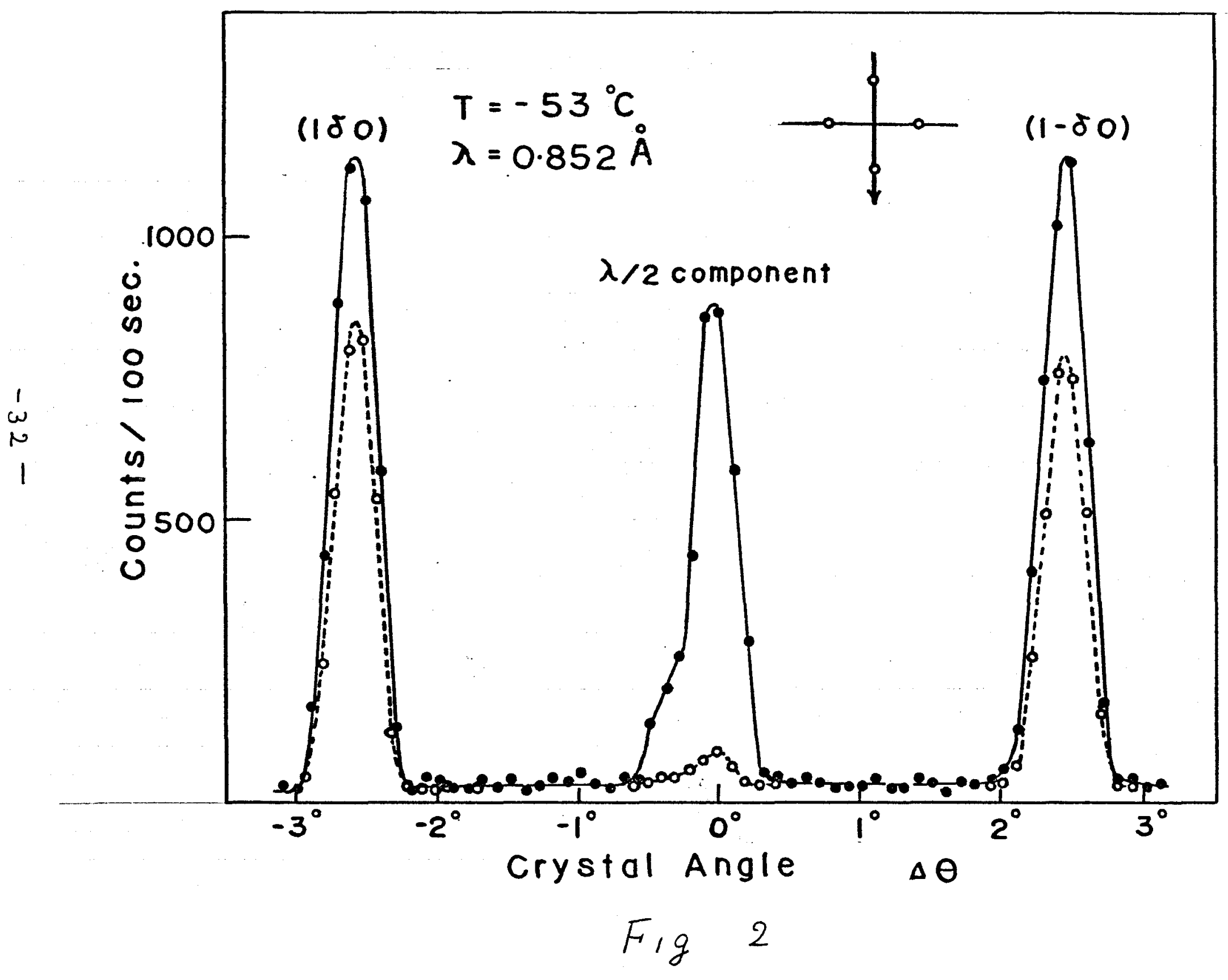




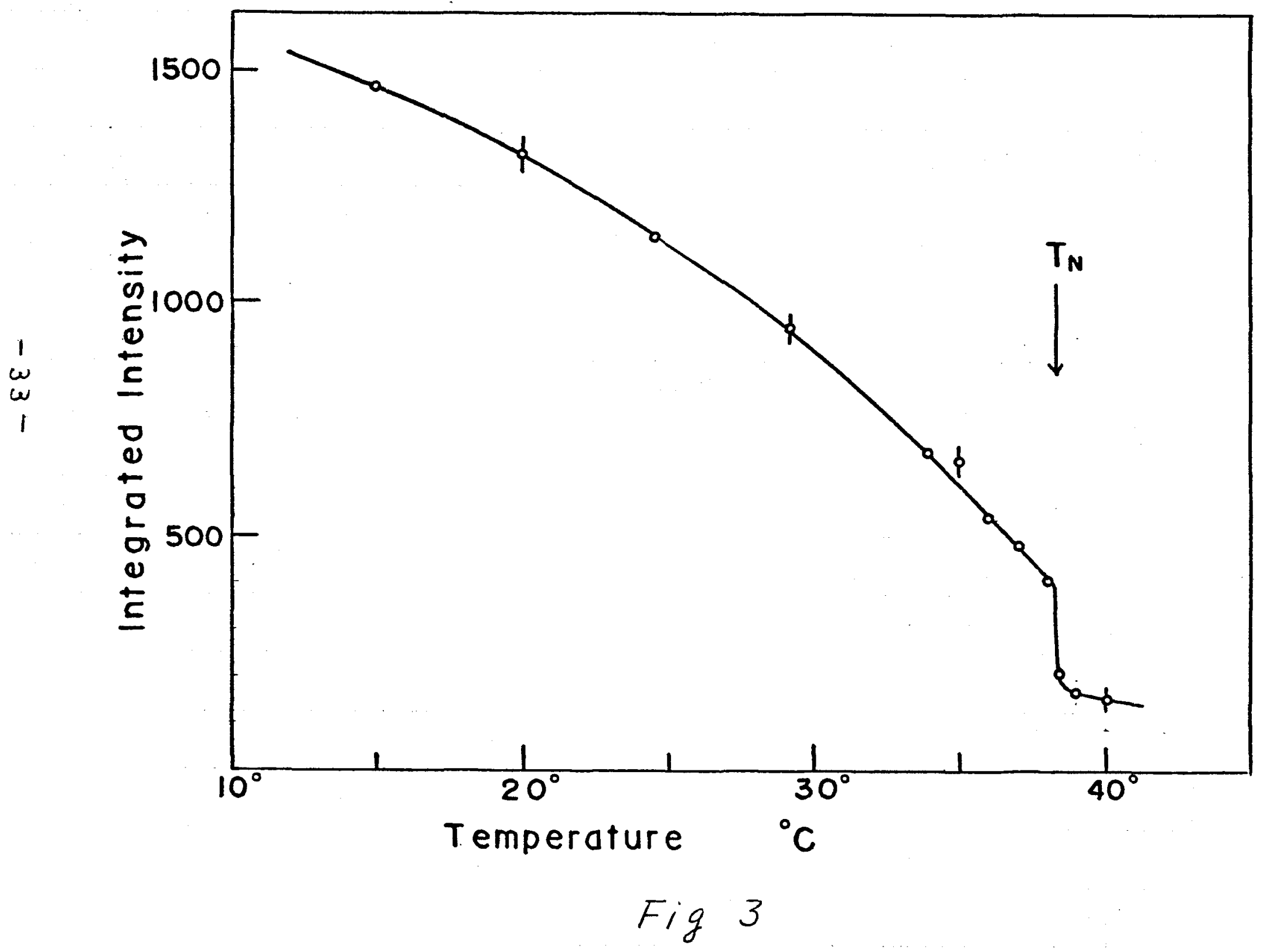




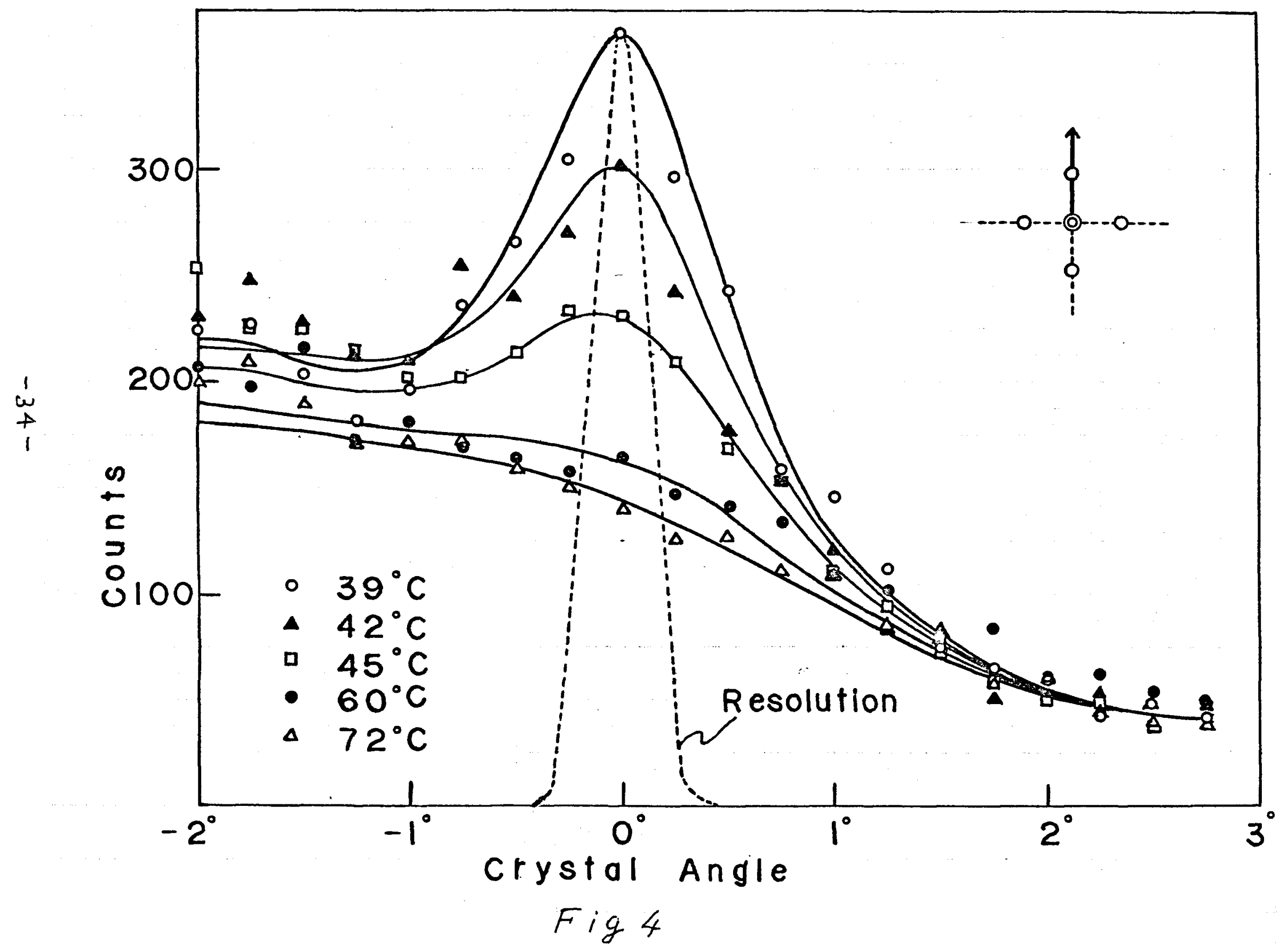




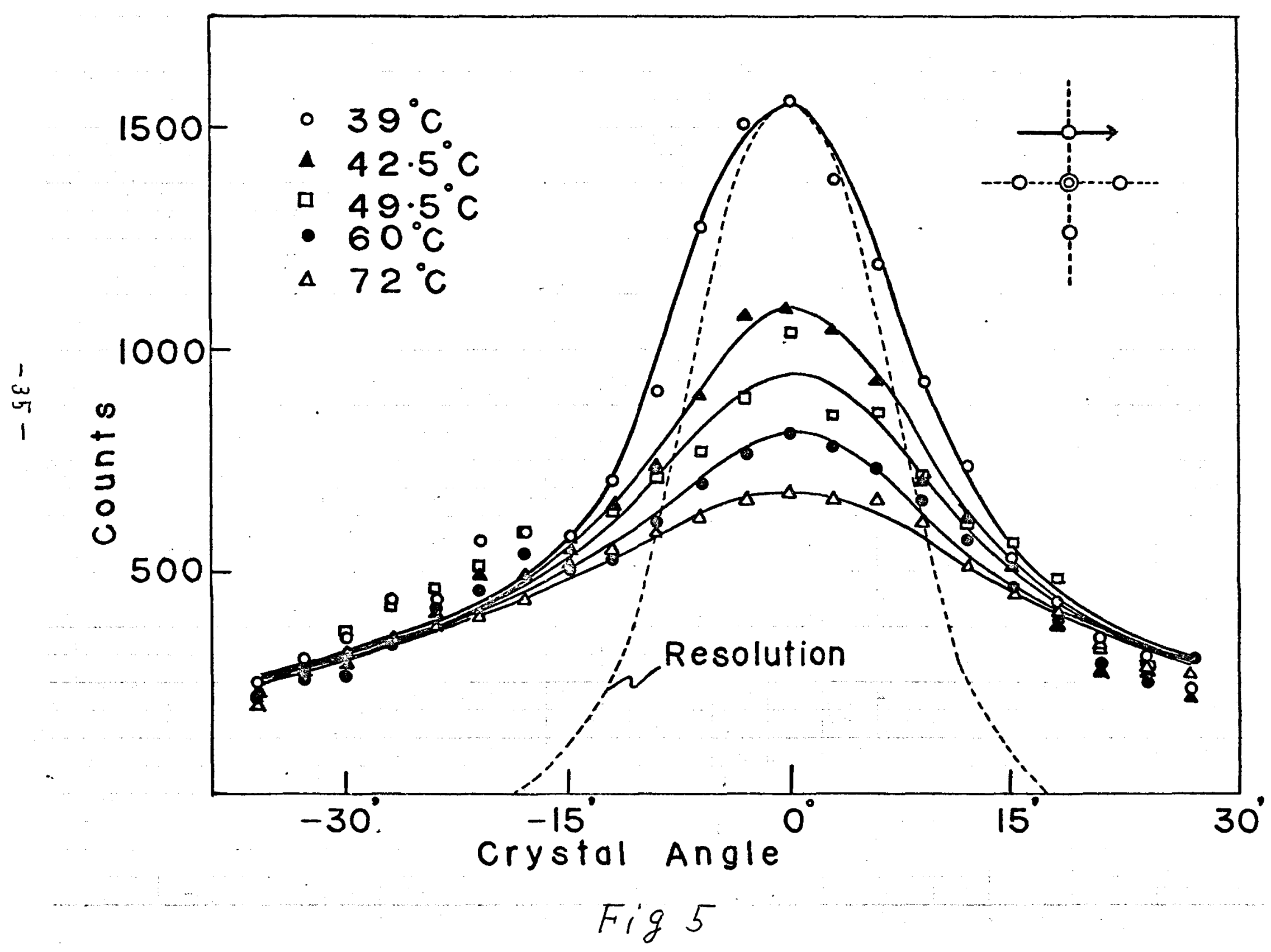




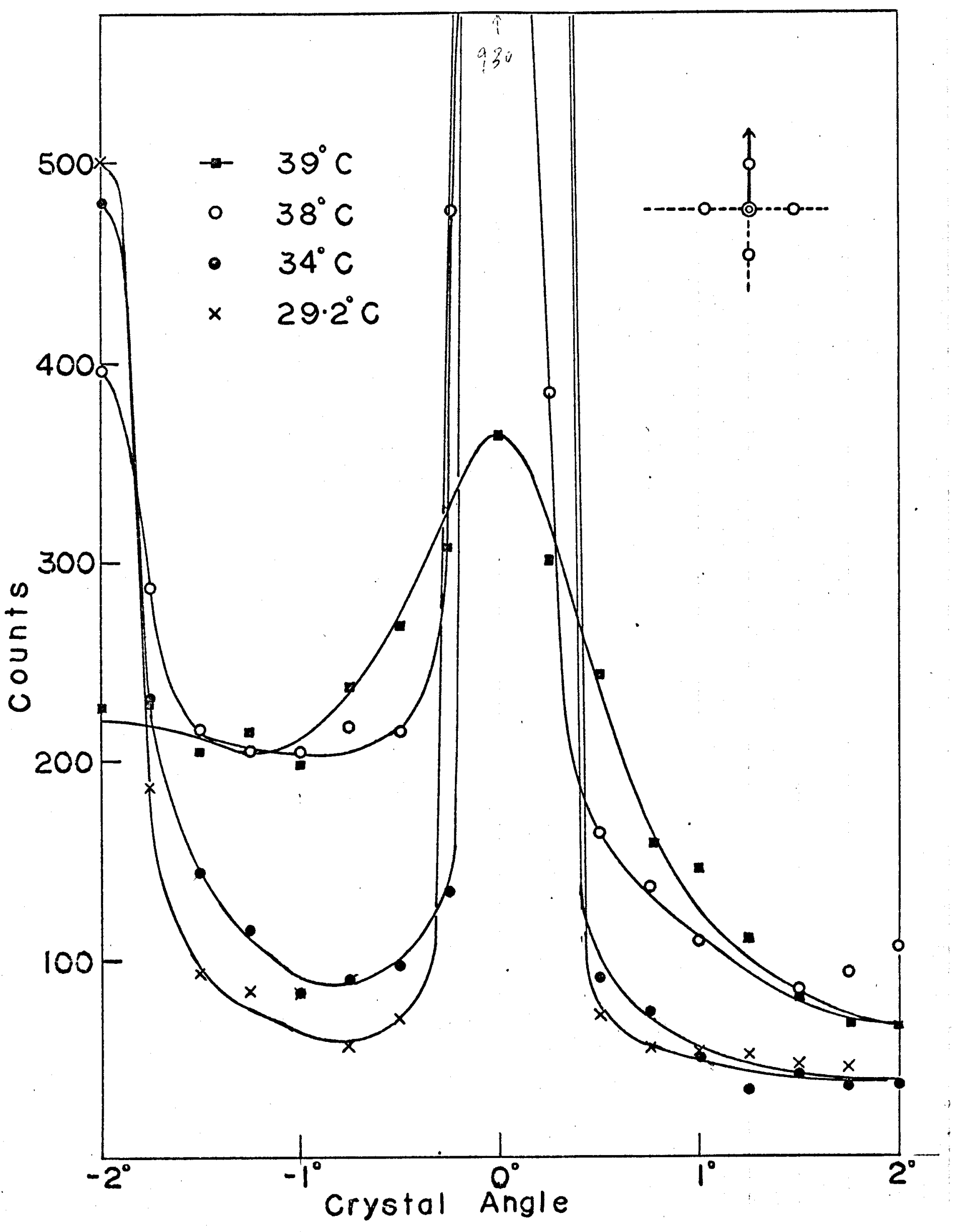

Fig 6. 


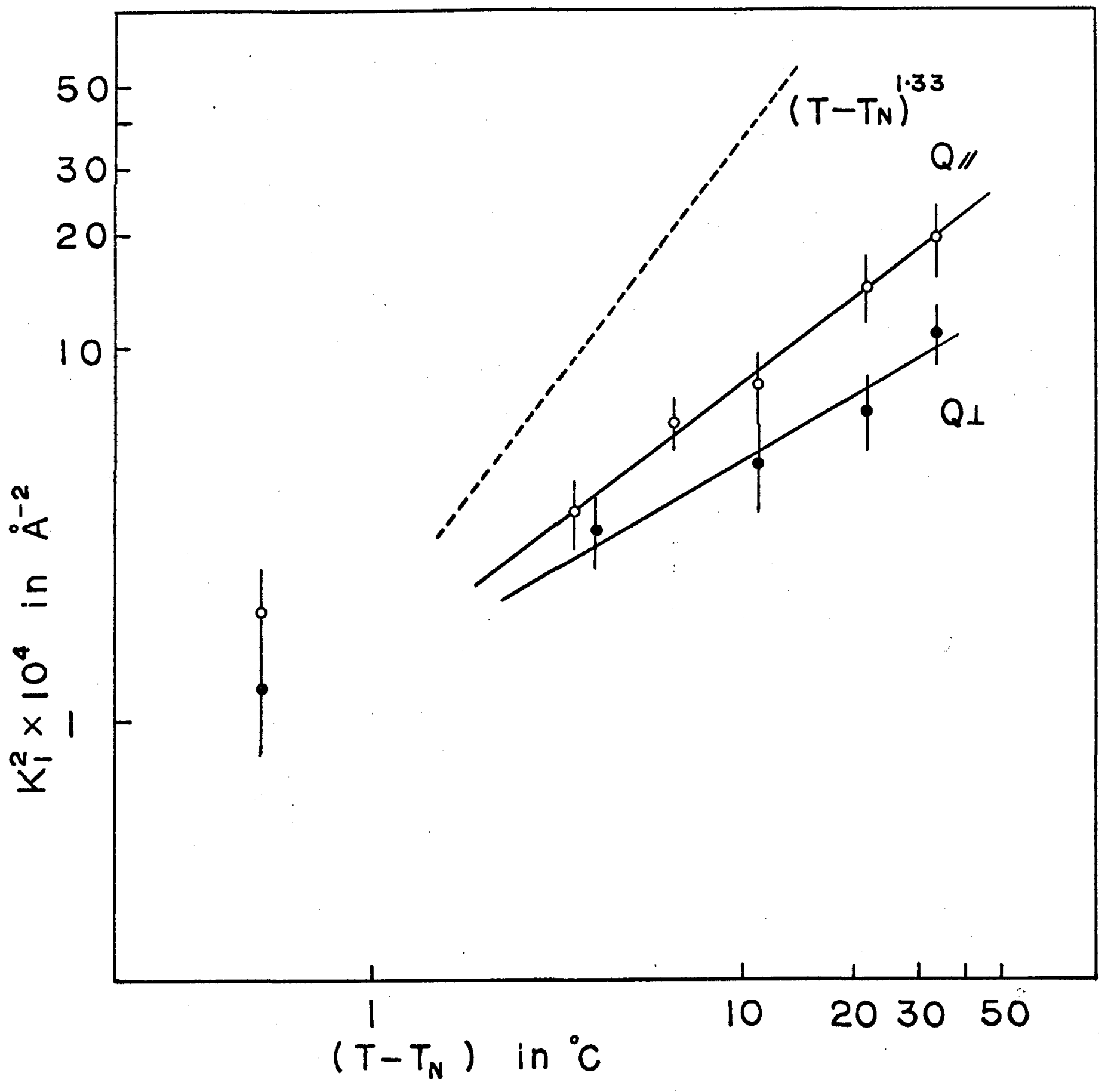

Fig. 7 


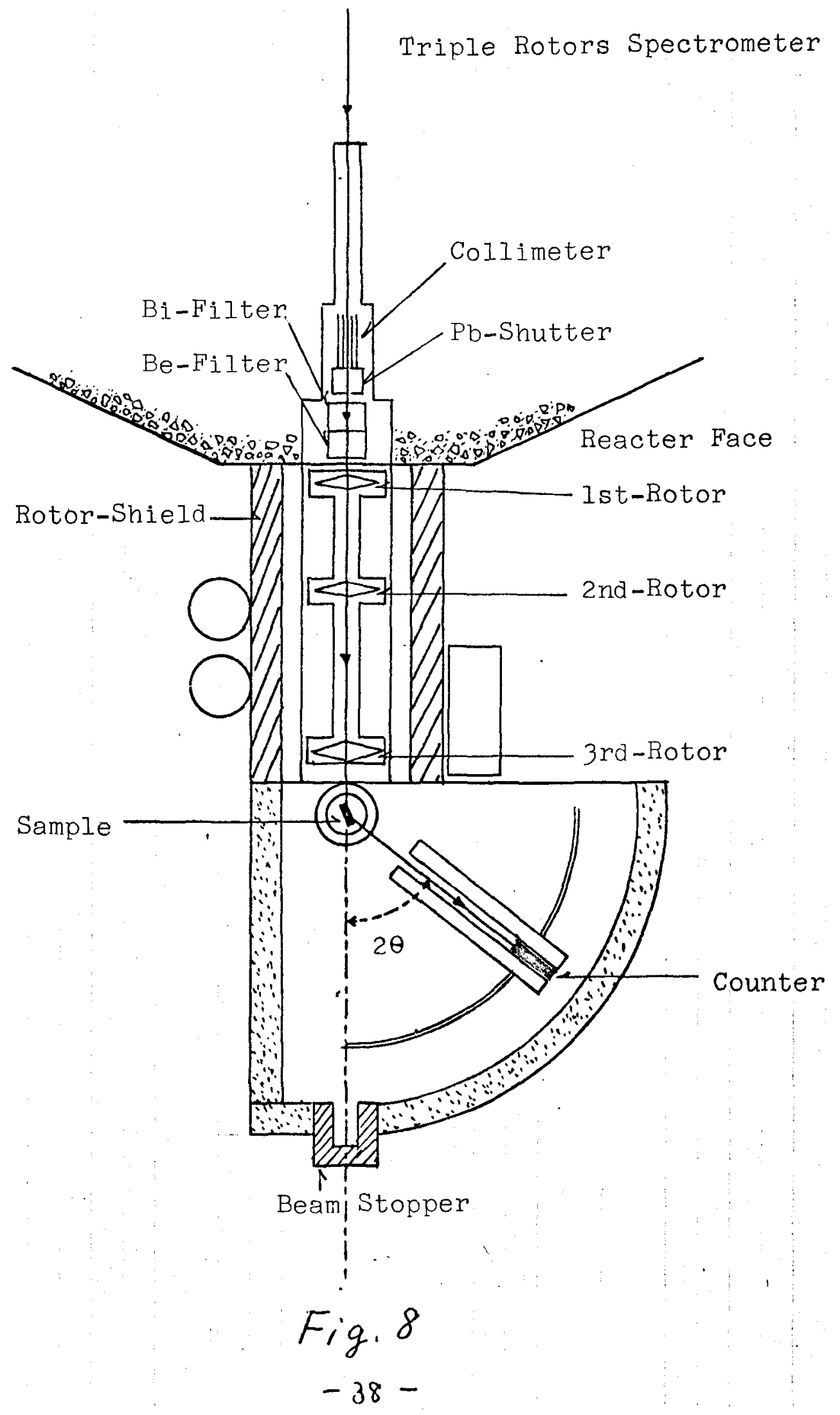




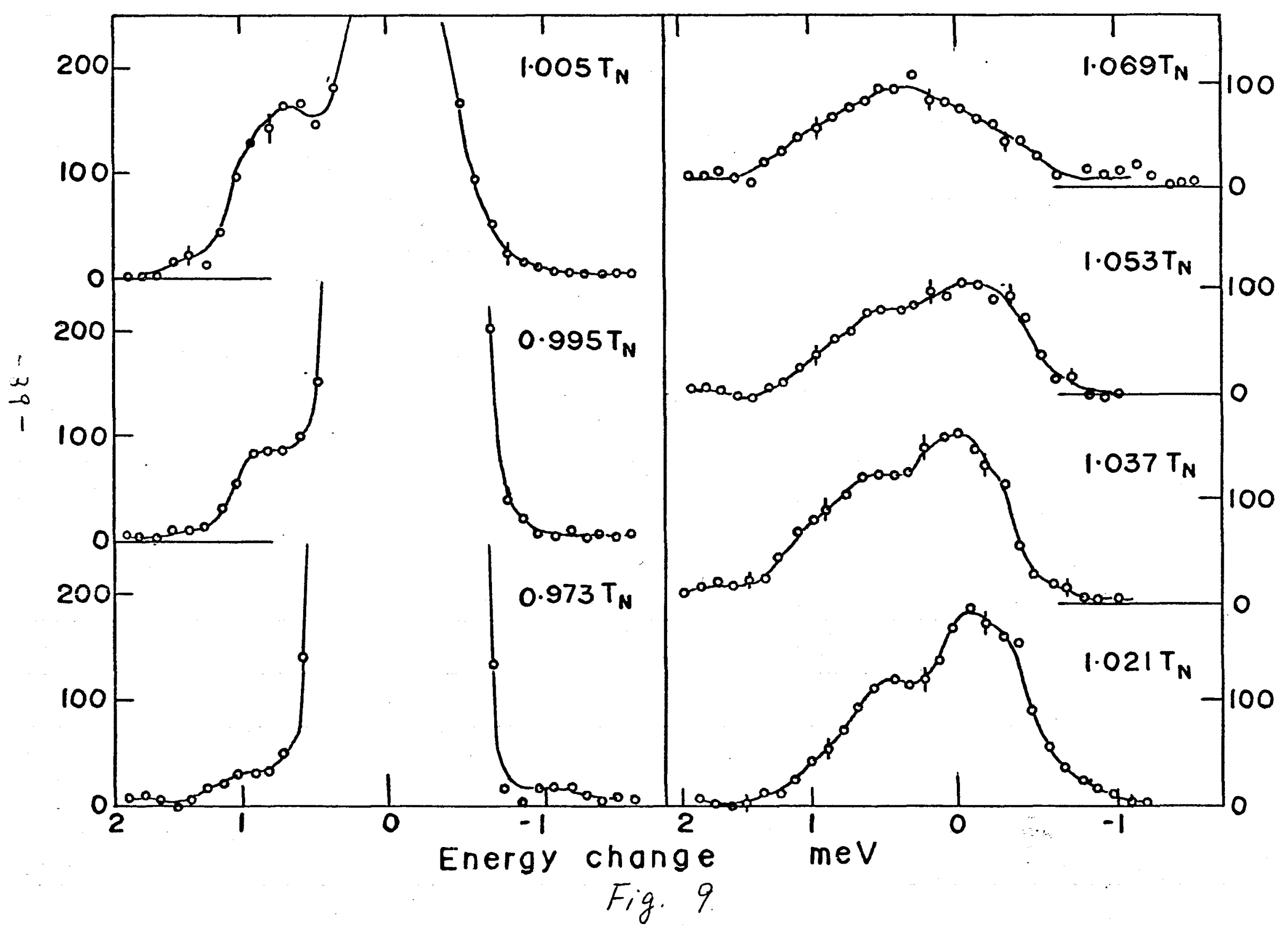




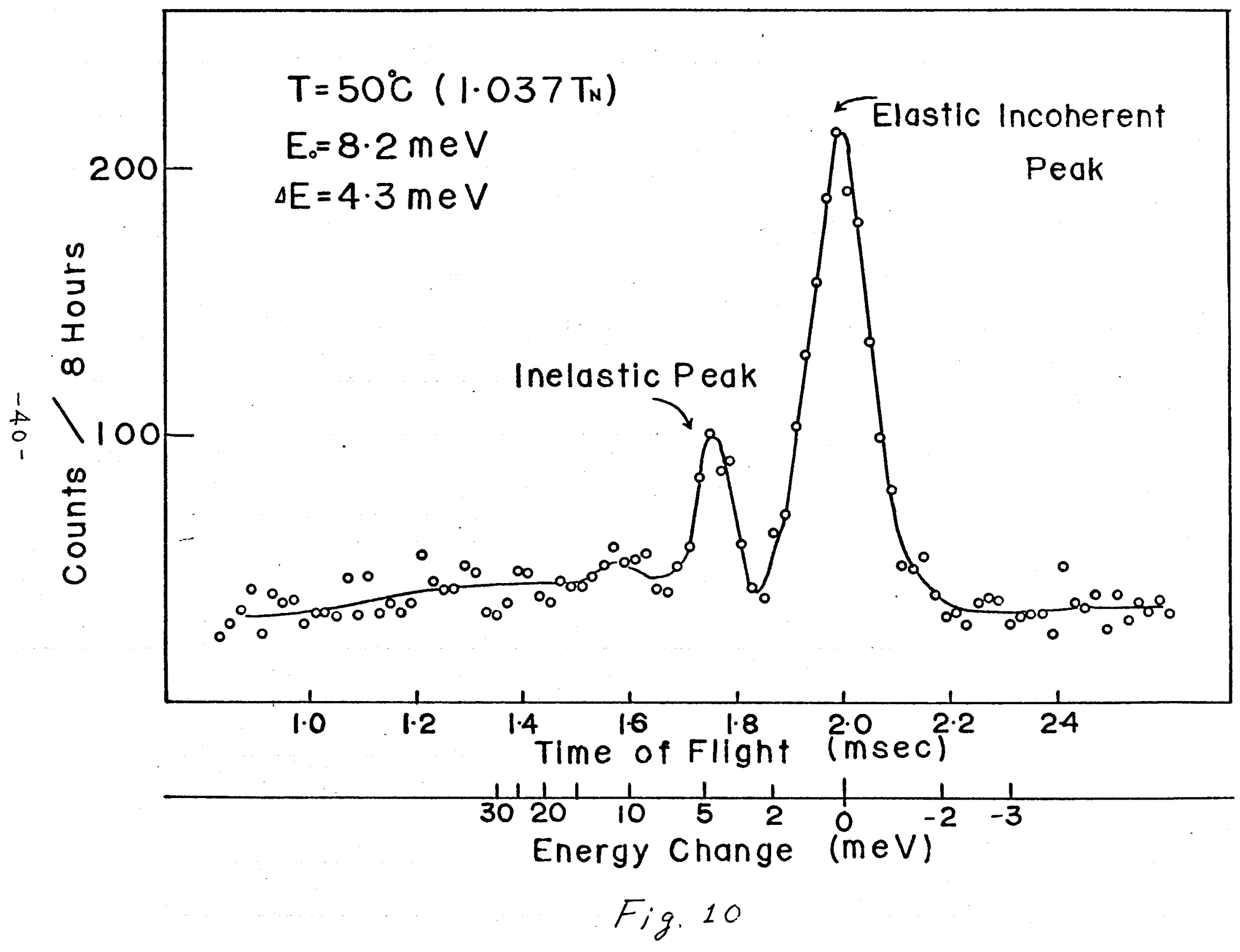



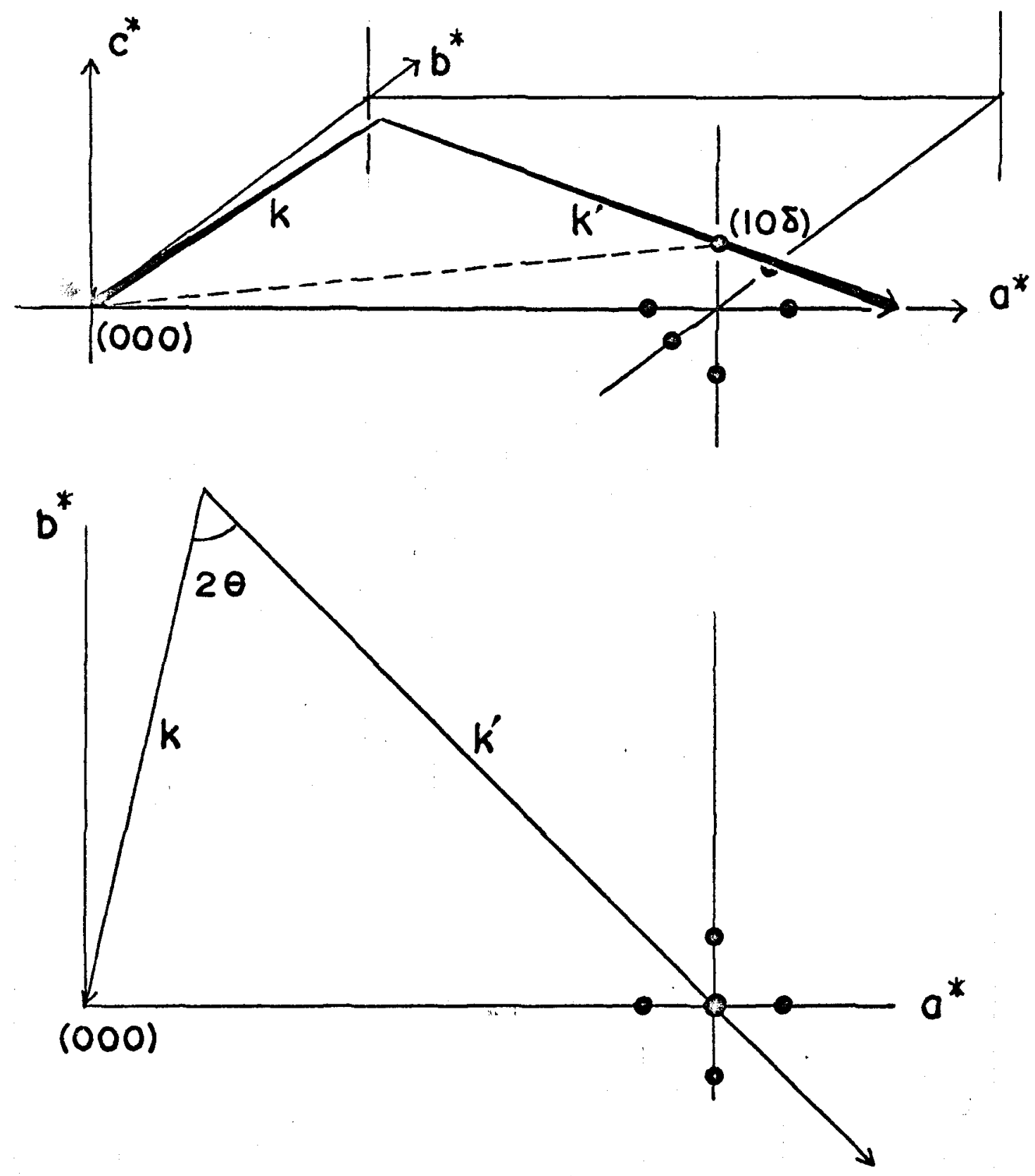

Fig. $11-a)$ 


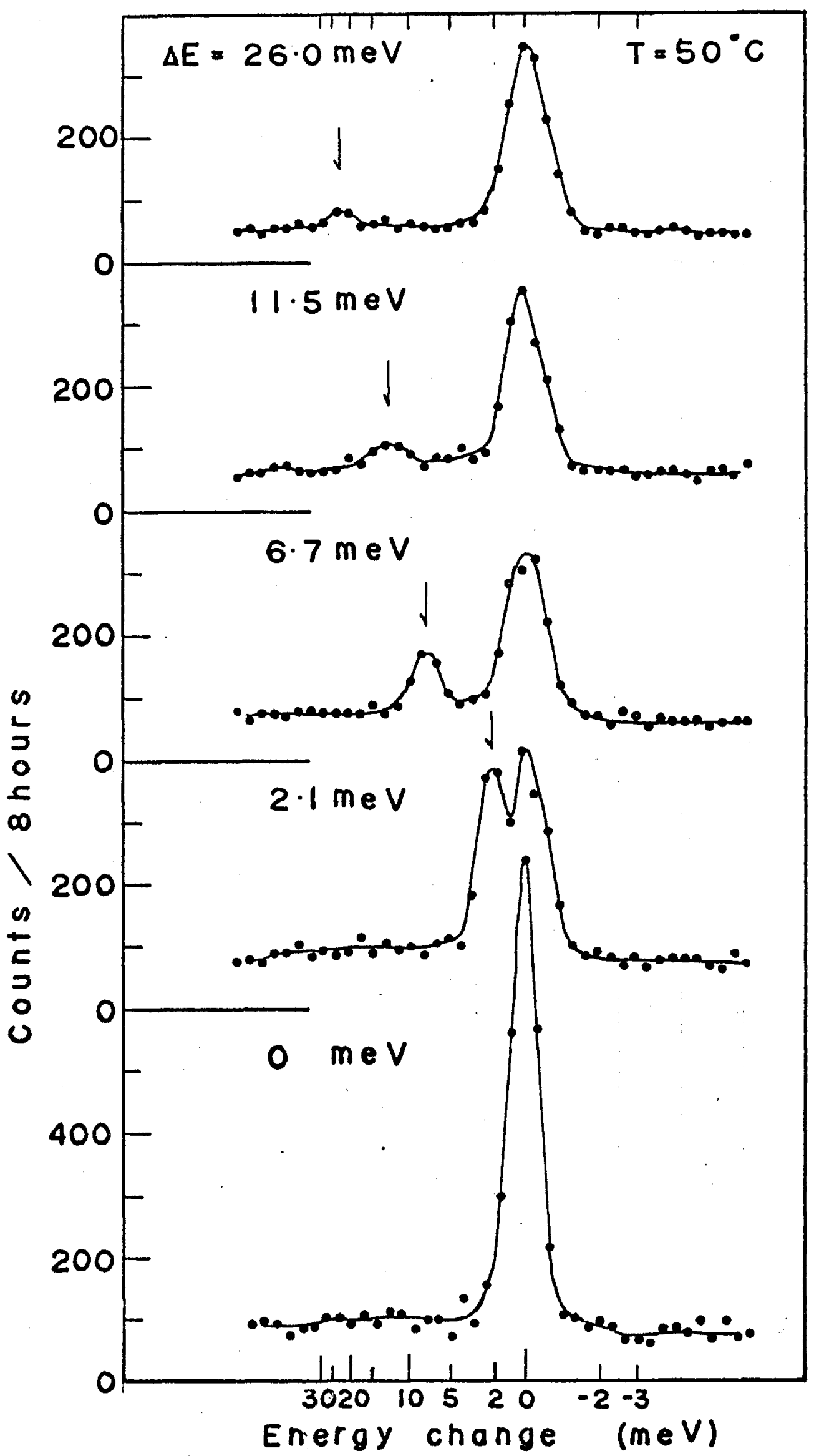

Fig. $(1-b)$ 


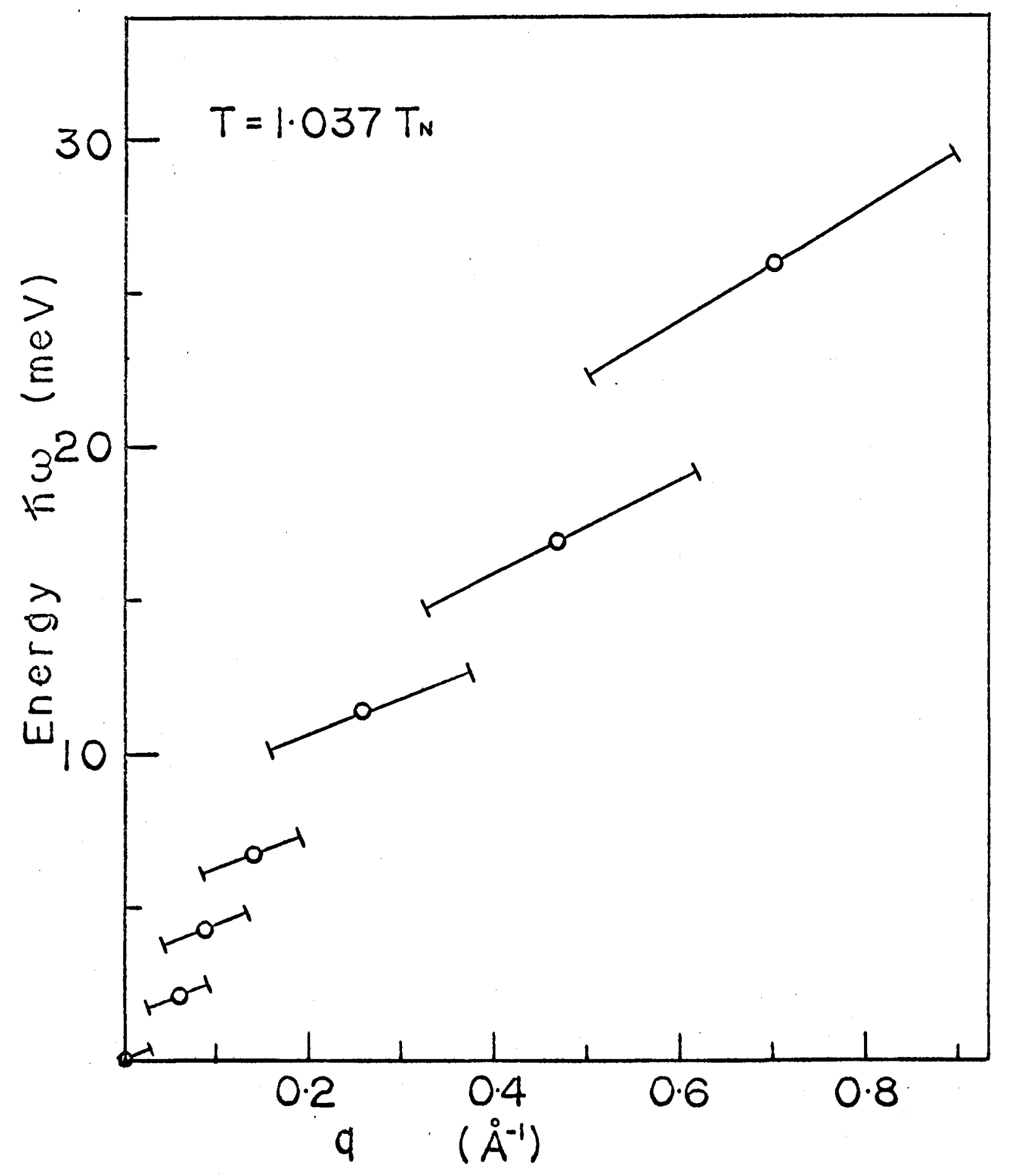

Fig. 12

$-43-$ 

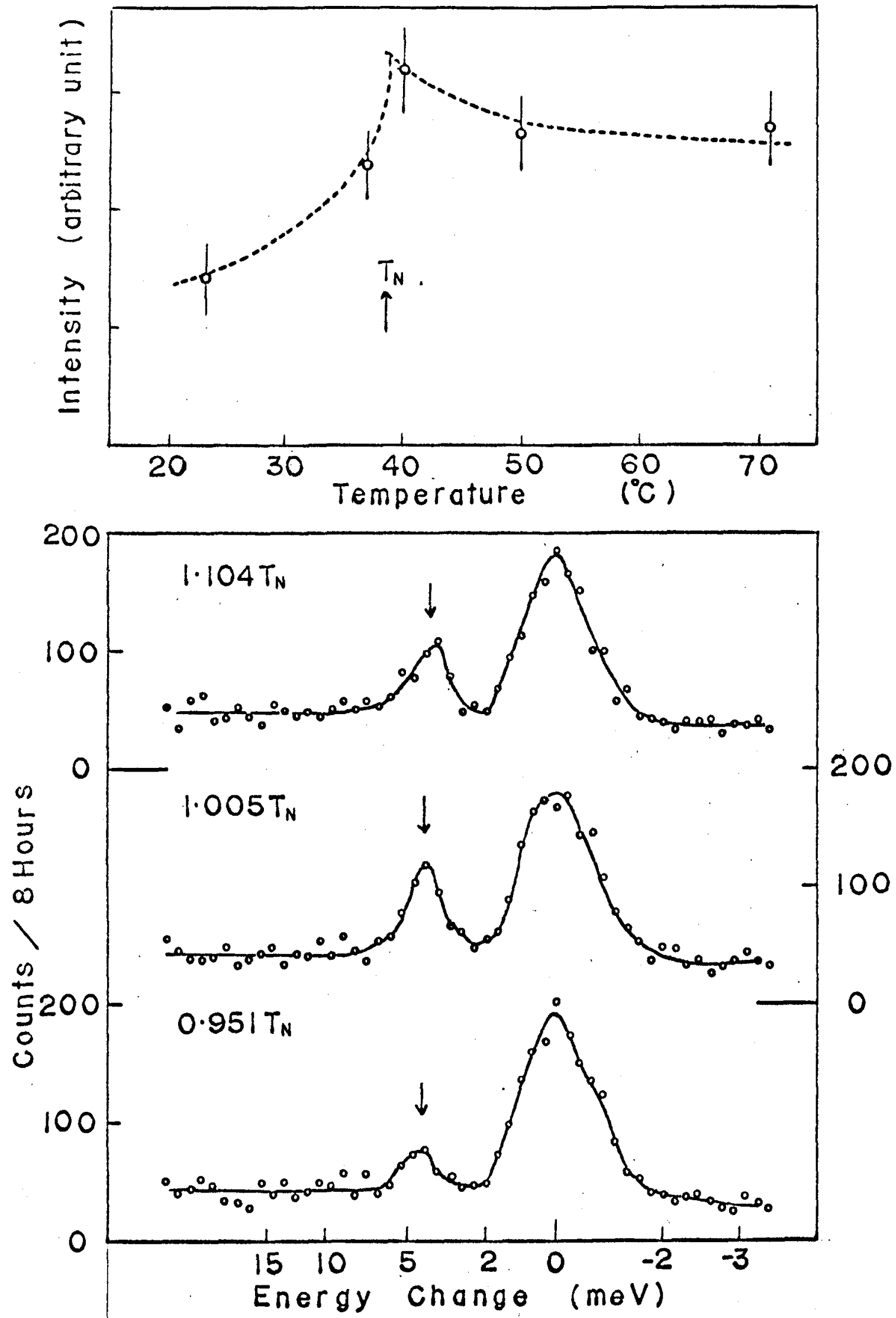

Fig. 13 